\title{
Role of vision in the optics learning process
}

\section{Marta Pesa, Elisa Colombo, Leonor de Cudmani}

Marta Pesa, Elisa M. Colombo, Leonor C. de Cudmani, "Role of vision in the optics learning process," Proc. SPIE 2525, 1995 International Conference on Education in Optics, (13 October 1995); doi: 10.1117/12.224028

SDIE Event: SPIE's 1995 International Symposium on Optical Science, Engineering, and Instrumentation, 1995, San Diego, CA, United States 
Role of vision in the Optics learning process

\author{
Marta Pesa - Elisa Colombo - Leonor C. de Cudmani \\ Instituto de Física - Facultad de Ciencias Exactas y Tecnología \\ Universidad Nacional de Tucumán \\ Av. Independencia 1800 - (4000) Tucumán - Argentina
}

\begin{abstract}
Most of the time, in the teaching of geometrical and physical optics, the visual system is not considered in the analysis of different phenomena. This situation is often reflected in the physic texts and is responsible of a limited comprehension of physic concepts involved. It is so natural for us to see that it is sometimes difficult for the physic teachers to give up intuitive conceptions about what and how we see.

The authors propose that the common sense is not enough. It is necessary to start from a scientific point of view considering contributions from diverse fields of analysis, avoing the tentation of reducing the behaviour of the vision system to a photgraphic camera. To see is much more than just to record images.

Vision seems effortless. Objects are recognised in the environment and actions are carried out accordingly. However, current thinking suggest that the task is performed by the cooperative action of many different modules, each with specific task. Theses modules may represent different parts of the brain, or different routines in a computer program.
\end{abstract}

This article stands out significant contributions from different approaches:

* Theory of information processing

* Artificial vision

* Computational vision

* Cognitive psychology theory of learning

These interdisciplinary contributions lead to the conclusion that the vision is an intended process. It is also able to make use of light propagation in the environment. This energy is processed constructing usefull visual representations and symbols. The conclusion is that the vision is a cognitive process.

Keywords: visual system - geometrical optics - physical optics - teaching and learning 


\section{INTRODUCTION}

Most of the time, in the teaching of geometrical and physical Optics, the visual system is not considered in the analysis of different phenomena. This situation, also reflected in the basic Physics texts, is often responsible of:

* a limited comprehension of some physical concepts involved like the concept of coherence or the concept of virtual image,

* some difficulties to understand, predict and explain experimental results with the optical systems at the laboratories,

* the lack of consciousness respect to the characteristics, functioning principles and limitations of the observer's eye as an optical detector.

This paper takes the students intuitive conceptions about vision as a starting point. Some examples about the persistence of these ideas and their negative influences on the learning are analyzed. The foundamental principles and notions of the visual systems, that we considered convenient to be included at the basic optics curricula for Physicist and Engineers, are stated. Finally, this article stands out significant contributions from different interdisciplinary approaches in order to get a correct conceptualization of the optical phenomena.

\section{PRECONCEPTIONS ABOUT VISION}

Recent science learning models enphasised (1) the important role of previous and intuitive knowledge in the construction of scientific conceptions. Furthermore, many scientific educational researches in the Optical area have brought to light those pre-conceptions showing that they are deeply anthropomorphic, centered on the perceptual phenomena, especially on the visual perception.

This behavior is not surprising from the genetical epistemology theory frame, which states the connections between the historical and psychological genesis of scientific conceptions. History of science shows that the "...questions about vision have been pondered even since philosophy began. Indeed, the Greeks modeled knowledge upon vision; they invested considerable effort in understanding how we come to know the visible world and how this knowledge may contribute to - or constitute - general understanding... Aristotle was interested in discovering how the eye actually works" (2).

If science teachers realize that it took a large and deep process of reflection (2000 years !!!) to overcome the precientific paradigm about vision and the nature of light and to 
formulate the first geometrical Optics models, it is undoubtedly reasonable to use the necessary time during instruction for the reconstruction of those conceptions (3).

The most critical problem during the Optics learning process is that the students hold a common sense conception about the meaning of vision. They are so familiar with seeing, that "...it seems that it takes a leap of imagination to realise that there are complex problems to be solved during vision." (4): the formation of real images "up side down" at the retina, the formation of a $3 \mathrm{D}$ image from a $2 \mathrm{D}$ intensity pattern, the objec recognition,"...and this is nothing short of a miracle" (4).

The prescientific conceptions about vision (3) (5) have several characteristics that deserve to be mentioned:

* Vision seems an effortless act. Objects are recognised in the environment and actions are carried out accordingly. However, scientific research suggests that the task is performed by cooperative actions of many different modules. These modules may represent different parts of the brain, or different routines in a computer program.

* Vision is a source of objective knowledge. The students sustain an empirist point of view when they consider perceptions as reliable base from which it is possible to apprehend the world "without disturbing it". However, there are certain evidence that suggest that "...there is much more when we see that what is discover by the eye ball" (6). What an observer perceives depends on his/her past experiences, his/her previous knowledge and his/her expectations. In this way there are qualitative differences between what is "seen" by an expert or what is "seen" by a "novice", for example, when they look at the same portion of the sky with a telescope.

* What it is not perceived, it is not conceived. In apparent contradiction with the intuitive conception of vision as "asceptic observation of reality" just mentioned, many students answers are strongly linked to perceptual sensations. In concordance with this idea, we have detected for example, that the students have firmly rooted the beleive that "the light emited by a source can travel only a finite distance $(1 \mathrm{~m}$ or $10 \mathrm{~m}$ or $1 \mathrm{~km}) "(5)$. Although this idea is wrong from a scientific perspective, it is founded in a phenomenological personal experience in everyday life. Scientific conceptions for light propagation as the ray model, or electromagnetic wave model, or photonic model, that hypothesized that light energy propagates without limits at vacuum, it is not directly perceived in the real world where the media are absorvent and dispersive.

* The eye behaves like a simple screen. Some students do not consider the eye as a complex optical system that behaves as an optical detector responsible of image formation. Even more, they do not consider the possibility of any processing of the incident light, neither the disturbing effects due to the system aberrations. They are not aware that each part of the eye is an extremely specialized structure. 
Effects such as spatial and temporal integration, threhold sensitivities, induced contrast, colour perception are only a few examples that even the formal instruction does not account for.

* The meaning of vision as generalization of inmediate experiences. Anything that we see turns inmediatly clear and in focus, and at the same time, all the objects sourrounding get blurred. This result could be the base of some surprisingly educational results (7) that have shown a deeply rooted implicit misconception associated to the permanence of the theory of light emanation: "mysterious rays of light come out from our eyes in straight line to make the objects visible" (8). This idea reminds the prescientific Greek conceptions. In fact it is a piece of evidence that the prescientific model could be explained because of its strong links to the everyday experience.

To sinthesized, it seems reasonable to think that as more complex aspects of the Optics are developed during the instruction, simultaneously conceptualizations more and more complex about the visual system aspects, have to be considered, until arriving to a model of it that detects, codifies, interprets, processes and re-constructs the external world (9).

\section{MISCONCEPTIONS OF VISION AND ITS EFFECTS ON LEARNING}

It has already been pointed out that teachers, the same as some usual textbooks on basic Optics, do not include the observer's eye (or any other kind of detector) in Optical systems. This means disregard one of the most perfect optical instrument, forgetting that the visual system is, most of the time, part of the experimental device for image formation, image processing, colour vision, the detection of patterns of light and darkness, etc.

* Difficulties detected in geometrical optics show the importance of considering the observer's eye as an optical system. We shall anlyze as examples two situations: the formation of real images with converging lens and the formation of virtual images with plane mirrors.

Real image formation. The fact of not including the obsever's eye makes it even more difficult for the student to understand the idea that a real image is formed without the help of a screen and in the case he/she accepts that possibility, to be able to decide about the more appropiate position for the eye to detect it. These data were collected primary through individual demostrations interviews, in which an investigator presents a series of tasks based on a experimetal demostration that the students observed in a system formed by a light source, a converging lens and a screen (Examples of some questions formulated during the interviews are: What happens with the image if you remove the sreen?; Where should you locate your eye if you want to see the image and the screen has been removed? (10) (11). 
During these interviews we detected a lack of understanding of the role of the eye exacerbated the problems the students had with the idea of observing a real image without a screen. Their lack of experience in directly viewing such image, coupled with the frequency with which they had seen one on a screen without analysing the diffusse reflection on the screen and the image formation at the retina, may have predisposed the misunderstandings.

Those students who recognized that the screen was not necessary to see the image often could not realize that the eye behaves at least as a converging system and that the divergent rays from the images point must enter the eye to form the image.

Virtual image formation. It is not obvious for the students, that the understanding of virtual image formation requires an understanding not only of how light reflects or refracts in a mirror or a lens, but also some reflexion about how an observer sees an image.

The interviews done by some researchers (12) presented tasks with plane mirrors (Example of a question formulated during the interviews is: If you replace the observer by a screen, can you see the image formed by the plane mirror on it?). Most of the answers reflected a lack of understanding of a fundamental conceptualization: both the formation and the perception of the image as inseparable processes, in other words, both the mirrors and the observer (or any other detector, like a camera) are necessary for the formation of a virtual image. In fact, the eye makes the diverging light reflected from the mirror, converge on the retina. Meanwhile typical diagrams made by most of the students show only one ray entering the eye, and are incomplete because they do not represent how the observer can both form and see the image (13).

Image inversion. It is usual to think that there must be a mechanism to invert the retinal image in same stage of the visual way suggesting the need of some kind of "internal eye" to see it. This is absurd. What the eyes do is to feed the brain with the information coded into neural activity changes of electrical impulses which by their codes and the patterns of brain activities represent objects. A similar process occurs with language: the letters and and words readen in a book, for example, have certain meanings, to those who knows the language. They affect the reader's brain, but they are not pictures. In other words, when we look at something, the pattern of neural activity represents the object and to the brain is the object. No internal picture is involved (8).

\section{* The study of interference phenomena requieres to consider the eye as a sensor system.}

Teaching experience shows that in the study of interference phenomena there are mistakes and confusions very settled that difficult the comprehension of physical phenomena involved. Different educational research (14) hypothesize that the foundamental reason is a strong internalization and permanence of the simplified model of light wave represented as a train of infinite lenght, amplitude and frecuency constant and linearly polarized. This idea 
implies to relate the concept of coherence only or basicaly with the emited source, considering the sun or an incandescent lamp, as incoherence sources and a laser as a coherence source, independing of the experimental system's dimensions and the detector's characteristics. This last point is the one we want to emphasize.

In fact, if we pay attention to the eye as a detector, two significative magnitudes are: the reaction time, related with the ability to discriminate interference patterns changing on time and the minimun sensitive area necessary to detect a luminous signal. In most of the interference experiments the eye is used as the detector but the students do not realize about this fact, and often they do not know that the order of magnitude of the reaction time is tenth of second and the order of magnitude of sensitive area is hundredth of milimeter.

Even more, the language keeps the wrong meaning. For example, when one talks about "interference patterns" instead of talking about "stable patterns of dark and brightness" when the sensor is "able" to differentiate, in time and in space, changes of intensity. At the same time, one talks about "absence of interference", instead of "a uniform intensity pattern", when the sensor "does not detect" the changes.

\section{* Understanding the concept of colour perception}

The white. One of the most usual mistakes connected with colour is considering "white" like a coulor that exists by itself, or even more, considering it as a sum of all spectral colours present in the visual spectrum. These are strong misconceptions. In the first case, the students do not consider that white is a perception resulting by the fact that the visual system is not able of discriminating the spectral components. The visual system in this case is not capable of seeing any colour, it sees "white" although there are sum of colours. In the second case, they are not aware that the perception of white can also be obtained by the sum of three primary colours or by the sum of two complementary colours.

Spatial integration. It is usual not taking into account how does a monochrome monitor actually work. Similar to the previous example the students think that "grey" is a real colour. In place of three types of phosphor, each emiting only a single hue integrated by the visual system, the students have an implicit and underlying "idea" that would imply a single phosphor emiting grey.

It is interesting to mention the results of some experiences that were performed with students in order to investigate knowledge about colour addition. Traying to explain differences between the "white" produced by the sum of three primary light on a screen and the "white" obtained on a mochrome monitor, it was surprising to register the following answers "white light comes out from the screen" but in the monitor situation "it is the eye which produces the integration". Obviously in both cases the important fact is that the visual system, in opposite to the auditive system, is not an analizer of modulated frecuency. 


\section{SOME BASIC CHARACTERISTICS ABOUT THE VISUAL SYSTEM}

All the situations already mentioned pointed out the importance of introducing the study of the foundamental aspects of the visual system and simultaneously looking for effective ways for the distructurization of intuitive conceptions. These are not easy tasks, it demands a critical and thoughtful attitude, to refomulate the curricula as well as the teaching strategies.

The introduction of the visual perception has to be gradual according to the optical phenomena studied. Starting with an objection to the common sense model of the eye as a screen, introducing real situations that bring to light its limitation, and evoluting gradually to the model that conceives the visual system as a camera till getting to the model of the information processing, and so on ...

From the point of view of the geometrical optics the visual system presents some interesting aspects to be analyzed. In fact, suppose we propose the students to design the mechanism of eye's refraction. First of all, as the image has to be projected on the retina, it has to be a real one. That means he/she has to design a system able to produce the same effect of a converging lens. Even more, it is necessary to get an image much smaller than the object because there is not enough room in the brain to reproduce the same magnitude orders of the external world. By this reason, the object has to be placed further than the double of the focal distance of the refracted surfaces in order to see small objects. Once the eyes' dimensions and the involved distances have been established some calculations allow to estimate the refractive charateristics of the eye.

At this point it is important to stand out that the eye presents some charateristics that are different from a camera. When the camera focus the image it changes the distance between the film and the lens while the eye focus the image changing the crystalline radius of curvature. Besides that, the cornea is responsible of most of the image focusing that is actually accomplished by the bending of light at its surface. In other words, the refractive system is formed by two components, the coarse adjust -in the cornea- and the fine one -in the crystalline. Another characteristic is that, in most of the photographic films, light acts in a permanent way; changes in the silver grains depend on the total amount of lighting energy received. It implies that the photographic film sums perfectly over time. Obviously the eye performs in a different way, it feeds the brain with a register from the visual scene time to time.

It should be realized that the response to light is not the same all over the retina. This is partly because there are two kind of photoreceptors, rods and cones, with different spectral and absolute sensitivities to light. They are not evenly distributed across the retina. By now, it should be apparent that the retina is much more than a film. Its structure differes from place to place, it undertakes a degreee of signal modification and it requires continual 
movement to operate. Functionally it is considered as part of the brain. If we considerer the eye as a detector it is neccesary to analize its spectral, temporal and spatial characteristics.

In addition, when the light arrives at the retina, the part of the eye responsible for converting the signal into physiological responses, there are some obstacles to the light. The retina is at the rear eyeball with the light-sensitive elements face in the wrong direction: they point inward, toward the brains, rather than outward, toward the light. As a result, to get out the information through the nerve fibers, there must be an opening in the back surface. This produces a blind spot in each eye that has to be frequently taking into account in optic research or teaching situations.

The retina actually works taking a discrete sample from the real excitation on the photoreceptors. At this point in the visual way, the information is transduced in electrical signals and it is pooled before travelling to the brain. The light that falls on part of the retina influences the neural responses of cell connected to other parts of the retina. The end result is to enhance the nervous system's reponses to the edges of the objects that it is perceiving, to afffect the brightness that is perceived, and to affect the perception of colour. In general, a region of brightness makes things around it seem darker. Similarly, dark regions make neighboring light regions seem even brighter. A patch of red will make neighboring regions look greener, and so on. To summarize, it is important to underly that the information sent by one photoreceptor is not independent of the neighboring responses and these relations are based of lateral inhibition. This is the most important functioning principle to explain a lot of perceptual illusions like Mach bands or induced contrast or induced colour.

Finally, the brain takes the signals from the eye and interprets them on the basis of past experience. How this is done is still a mystery, It is in this area that psychology and physiology meet.

\section{5.- CONTRIBUTIONS FROM DIFFERENT APPROACHES}

Vision is a process to solve problems. This task is much harder than might appear. Indeed, scientists still do not know how it is done. They are making progress, building each time more and more complex models, but they still have a long way to go.

Using the eye as a detector an intensity pattern is registered at the retina. From this point all other information must be built up in order to recognize objects, be aware of their positions in space, their sizes, textures and motions.

This process of transforming and interpreting sensory information is a complex one, and involves a considerable variety of processing mechanisms. At the very least, perception depends upon the physiological systems associated with each sensory modality, together with central brain processes which integrate and interpret the output from these 
physiological systems. Nor surprisingly, major contributions to our knowledge of perception have come from advances in both physiology and psychology. There is also increasing interest within the field of artificial intelligence in the possibility of computer simulation of certain aspects of perception (15).

The vision process intents to explain several questions:

* how does the visual system form an image on the retina?

* how is it possible to draw out from the retina the information necessary to reconstruct the external world?

* how the information is processed?

* what is the nature of the internal representations necessary to reconstruct the reality?

The research problem then is to dicover those processes that can get from an intensity array to representations that support highly flexible visual cognition. Template matching is the simplest of all possible models for recognizing patterns (16). Recognition is accomplished by matching the external signal against the internal template. However, attemps to recognize objects by direct template matching, have had little success. This model turns critical because it does not take into account that vision is conceptually driven. That is "the processing starts with conceptualization of what might be present and then looks for confirming evidence, biasing the processing mechanisms to give the expected result..." (16). Vision is evidently linked to cognition. It depends in complex way on the perceivers expectations and previous knowledge as well as the information available in the stimulus itself.

Besides that, things do not always appear as they actually are. What we see (or hear) is not always what is. Often the perceptual system makes errors, sometimes we see illusions, sometimes the perceptual system requires time to recover from prolonged stimulations, sometimes information that is not present in the scene must be added. An image could be ambiguous because of a lack of relevant information or a surplus of irrelevant data or it can also be ambiguous because of the existence of several different ways of constructing a meaningful interpretation, sometimes clusters of similar shapes or breaks in a repetitive pattern stand out as figures, sometimes there is a combination of the information conveyed by the local features with the considerations of the global and contextual interpretations. All these facts require more and more complex models.

In fact, Marr's model is one of the most successful attempts. This model gets some kind of integration from psychological, physiological and artificial intelligence viewpoints. Marr's (17) starting point was that there are three levels of explanations for visual perception. The top level is the computational level, which relates to the purpose of perception, and the bottom level is the hardware level. At an intermediate level, there is a algorithmic level which is concerned with the detailed precesses involved in perception. He proposed that the 
processes involved in vision produces a series of representations or descriptions which provide increasingly detailed information about the visual environment.

As a conclusion it can be say that there is not just one model that explains vision. As in Physics, there are different aproaches, and the models to be used depend on the problems that need to be solved.

\section{6.- REFERENCES}

1 - Posner G., Strike K., "A revisionist theory of conceptual change" - In "Phylosophy of Science, Cognitive Psychology and Education Theory and Practice" Editors: Duschl R. and Hamilton R. - State University of New York Press, Albany, New York, 1993

2 - Gardner H., "The mind's new science", Basic Books, Inc., Publishers, New York, 1987

3 - Guesne E., "Light" - In "Children's ideas in science", Chap. 2, Editors: Driver R., Tiberghien A., Guesne E., Open University Press, England, 1985

4 - Gregory R., "Eye and Brain - Psychology of seeing", Weidenfeld and Nicolson Press., London, 1990

5 - Pesa M., Cudmani L. C. de, Bravo S., "Formas de razonamiento asociadas a los sistemas preconceptuales sobre naturaleza y propagacion de la luz" Accepted to be published, Caderno Catarinense de Ensino de Fisica, Brasil, 1995

6 - Chalmers A., "Que es esa cosa llamada ciencia?", Siglo XXI Ed., Buenos Aires, 1988

7 - Pesa M., Cudmani L., C. de, "Paralelismo entre modelos historicos y precientificos en la ensenanza de la Optica" Caderno Catarinense de Ensino de Fisica, Vol 4 (1), Brasil, 1994

8 - Begbie H., "La vision y el ojo. Una introducción a la percerción visual" Editorial Universitaria de Buenos Aires, 1969

9 - Falk D., Brill D., Stork D., "Seeing the light", Harper \& Road Publishers, New York, 1990

10 - Pesa M., Cudmani L. C. de, Bravo S., "Do teachers "help" to strengthen misconceptions? Some reflections about the image formation with converging lenses" Sent to be published, 1995

11 - Goldberg F., Bendall S., Galili I., "Lenses, pinholes, screens and the eye" Physics Teacher, Vol. 29, 1986

12 - Goldberg F., McDermott L, "Student difficulties in understanding image formation by a plane mirror" Physics Teacher, Vol. 24, 1986

13 - Galili I., Goldberg F., Bendall S., "Some reflections on plane mirrors and images" Physics Teacher, October, 1991

14 - Leonor Colombo de Cudmani et al., "Optica Física Básica. Estructurada alrededor del concepto de coherencia luminosa", Universidad Nacional de Tucumán, Publicación Nro. 1441,1990

15 - Eysenck M., Keane M., "Cognitive psychology", Lawrence Erlbaum Associates, Publishers, London, 1991 
16 - Lindsay P.H., Norman D.A., "Information processing. An Introduction to Psychology", Academic Press, London, 1977

17 - Marr D., "Vision - A computational investigation into the Human Representation and Processing of Visual Information", Freeman and Company, New York, 1982 